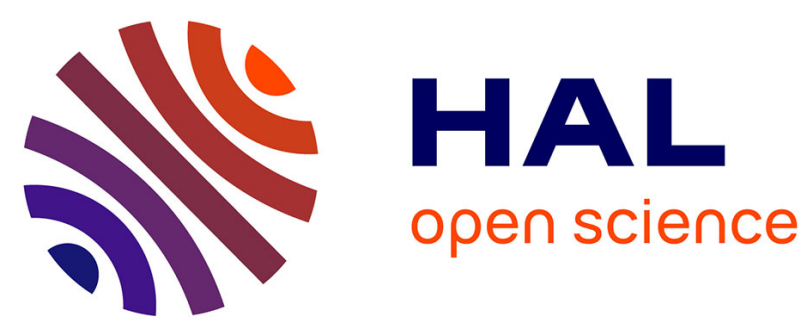

\title{
Numerical and experimental study of Boussinesq wall horizontal turbulent jet of fresh water in a static homogeneous environment of salt water
}

\author{
Aicha Belcaid, Georges Le Palec, Abdeslam Draoui
}

\section{To cite this version:}

Aicha Belcaid, Georges Le Palec, Abdeslam Draoui. Numerical and experimental study of Boussinesq wall horizontal turbulent jet of fresh water in a static homogeneous environment of salt water. Journal of Hydrodynamics, 2015, 27 (4), pp.604-615. 10.1016/S1001-6058(15)60522-4 · hal-01459167

\author{
HAL Id: hal-01459167 \\ https://hal.science/hal-01459167
}

Submitted on 13 Mar 2019

HAL is a multi-disciplinary open access archive for the deposit and dissemination of scientific research documents, whether they are published or not. The documents may come from teaching and research institutions in France or abroad, or from public or private research centers.
L'archive ouverte pluridisciplinaire $\mathbf{H A L}$, est destinée au dépôt et à la diffusion de documents scientifiques de niveau recherche, publiés ou non, émanant des établissements d'enseignement et de recherche français ou étrangers, des laboratoires publics ou privés. 


\title{
Numerical and experimental study of Boussinesq wall horizontal turbulent jet of fresh water in a static homogeneous environment of salt water"
}

\author{
BELCAID Aicha ${ }^{1,2}$, LE PALEC Georges ${ }^{1}$, DRAOUI Abdeslam ${ }^{2}$ \\ 1. IUSTI Laboratory, UMR 7343, Aix-Marseille Université, Technopôle de Château-Gombert, 5 rue Enrico Fermi, \\ 13453 Marseille Cedex13, France \\ 2. Energy Laboratory, Research in Energy and Thermal Transfer Team, Abdelmalek Essaâdi University, Faculty \\ of Sciences and Techniques, Tangier, Morocco,E-mail: belcaid_aicha@yahoo.fr
}

\begin{abstract}
This paper investigates a numerical and experimental study about buoyant wall turbulent jet in a static homogeneous environment. A light fluid of fresh water is injected horizontally and tangentially to a plane wall into homogenous salt water ambient. This later is given with different values of salinity and the initial fractional density is small, so the applicability of the Boussinesq approximation is valid. Since the domain temperature is assumed to be constant, the density of the mixture is a function of the salt concentration only. Mathematical model is based on the finite volume method and reports on an application of standard $k-\varepsilon$ turbulence model for steady flow with densimetric Froude numbers of 1-75 and Reynolds numbers of $2000-6000$. The basic features of the model are the conservation of mass, momentum and concentration. The boundaries of jet body, the radius and cling length are determined. It is found that the jet spreading and behavior depend on the ratio between initial buoyancy flux and momentum, i.e., initial Froude number, and on the influence of wall boundary which corresponds to Coanda effect. Laboratory experiments were conducted with photographic observations of jet trajectories and numerical results are described and compared with the experiments. A good agreement with numerical and experimental results has been achieved.
\end{abstract}

Key words: wall jet, turbulence, Boussinesq approximation, $k-\varepsilon$ model, buoyancy, cling length

\section{Introduction}

A wall turbulent jet is an effective means to promote high rates of heat and mass exchange especially in the near field. This jet configuration has been widely used in coastal sewage disposal projects. Indeed, it is necessary to ensure rapid mixing of the jet with the ambient fluid to minimize the impact on the marine environment and prevent a return flow of the jet towards the injection source. On this point, buoyant wall jets have a larger mixing zone than free jets and therefore it is possible to improve the initial dilution compared to the later. For a free turbulent jet, the expansion rate is the same in each normal direction to its centerline trajectory. However, it is not the case for wall turbulent jet.

\footnotetext{
* Biography: BELCAID Aicha (1985-), Female, Ph. D., Trainer and framer
}

When a wall turbulent jet is injected from sources of buoyancy and momentum, both effects of buoyancy and wall boundary are taken into account. In this case, the jet clings to the wall (obstacle) under the Coanda effect (definition in Section 1.1). The difference of pressure causes the jet cling to the wall and slip for some distance, called the cling length, before it leaves the floor. The Coanda effect of buoyant wall jets can increase the mixing zone between jet fluid and ambient so that the pollution in the near field can be effectively diluted. Singh and Ramamurthi ${ }^{[1]}$ investigated experimentally a gas turbulent plane wall jet. They used PIV technique to determine the conditions for which the jet would be affected by Coanda effect. Sharp and Vyas ${ }^{[2]}$ gave the relations between the cling length and densimetric Froude number through experimental research and theoretical analysis. Combining with Sharp's experimental data and theoretical results, Huai et al. ${ }^{[3]}$ applied the realizable $k-\varepsilon$ model to simulate horizontal buoyant wall jet and they gave the results of cling length, centerline trajectory and tem- 
perature dilutions at several sections. Li et al. ${ }^{[4]}$ presented a LES study of the flow features in the near region of a turbulent circular wall jet discharging from a nozzle into a static ambient. They determined the mean and turbulent velocity characteristics in the near region.

In the current study, the aim is to build on these past observations by both numerical simulation and experimental investigation to analyze a Boussinesq turbulent horizontal buoyant wall jet of fresh water into a static and homogenous ambient of salt water with different values of salinity. Modeling is based on a Reynolds Averaged Navier Stokes finite volume method. The standard $k-\varepsilon$ turbulence model for steady flow has been adopted with buoyancy related source terms in the momentum and turbulence governing equations. To validate numerical results, a set of laboratory experiments was conducted to observe photographically the jet boundaries, centerline trajectories and radius, and cling length over a range of initial conditions (initial jet velocity, nozzle diameter of injection and initial density difference between jet and ambient fluid of salt water). Numerical and experimental results are compared in order to validate the theoretical analysis.

\section{Mathematical and numerical models}

\subsection{General hypothesis}

We consider here mixed convection from a circular section source of fresh water placed tangentially on a horizontal adiabatic wall which is embedded in a static and homogenous ambient of salt water. At the source, the jet is characterized by the nozzle diameter, the injection velocity (supposed uniform), and the initial density gradient between the jet and the ambient fluid. The flow is tridimensional, turbulent and stationary. Since the domain temperature is assumed to be constant, density gradients are function of the mass concentration (salinity) only.

The applicability of Boussinesq approximation ${ }^{[5,6]}$ is limited to an initial fractional density $\Delta \rho_{0} / \rho_{a}$ of $0.05^{[7]}$ where $\rho_{0}$ is the density at the source and $\rho$ $\mathrm{a}$ is the ambient density, but to generalize this approximation Swain et al. ${ }^{[8]}$ suggested a condition of $\Delta \rho_{0} / \rho_{a} \ll 1$ for jets of light fluid injected into an ambient environment of high density. In these cases where Boussinesq approximation is invalid, a density equation must be taken into consideration.

For a wall flow, Coanda effect is usually adopted. The Coanda effect is the tendency of a fluid to cling to a surface that is near to an orifice from which the fluid emerges $^{[9]}$. Some authors ${ }^{[10]}$ consider both pressure difference and entrainment phenomena as examples of Coanda effect. Indeed, when two laminar jets injected from source of momentum (and/or buoyancy) are placed side by side, they tend to bent towards each other as a result of pressure difference. However, when the flow is turbulent, the jets bend due to the entrainment of ambient fluid. The present work will be based on the hypothesis that the attachment of turbulent round wall jet is caused by the momentum flux related to entrainment phenomena near the adjacent wall.

\subsection{Entrainment concept}

The entrainment assumption assumes that the velocity of ambient fluid entrained into the plume body is proportional to the mean centerline velocity of the plume. Thus, determining forced plume behavior leads to specify the rate of entrainment. The theory of plumes set out by Morton in 1956 assumed a constant coefficient of entrainment $\alpha$. However, this latter cannot be a universal constant ${ }^{[11]}$. For forced plume which should behave more like a momentum plume near the source and like a plume at large distances, Fan and Brooks ${ }^{[12]}$ used a value of 0.082 for $\alpha$ and their analysis agreed well with experimental data.

Based on the results of previous studies, Houf and Schefer ${ }^{[13]}$ have demonstrated that the local rate of entrainment for buoyant plume (forced plume) increases as plume leaves the region dominated by momentum and enters a region dominated by buoyancy forces. Thus, the entrainment coefficient is related to the rate of entrainment $E^{[14]}$ by

$E=2 \pi b \alpha U$

where $b$ is the width plume and $U$ is the centerline velocity. The local rate of entrainment $E$ is written in function of two components of entrainment, $E_{\text {mom }}$ due to plume momentum and $E_{\text {buoy }}$ due to buoyancy:

$E=E_{\text {mom }}+E_{\text {buoy }}$

$E_{\mathrm{mom}}=0.282\left(\frac{\pi d^{2}}{4} \frac{\rho_{0} U_{0}^{2}}{\rho_{a}}\right)^{1 / 2}$

$U_{0}$ is the plume velocity at the source (the nozzle) and $d$ is the local diameter of the plume.

$E_{\text {buoy }}=\frac{2 \pi U b a}{F r_{1}} \sin \theta$

where $F r_{1}$ is the local Froude number defined by

$F r_{1}=\frac{U^{2} \rho_{0}}{g d\left(\rho_{\infty}-\rho\right)}$ 
As the local Froude number decreases, the $E_{\text {buoy }}$ term begins to contribute and the buoyancy effect becomes more important.

In Eq.(4) it appears that the constant a, with a determination based on experiments developed by ${ }^{[15]}$, is related to densimetric Froude number $F r$ by:

$$
a=17.313-0.1166 \mathrm{Fr}+2.0771 \times 10^{-4} \mathrm{Fr} \text { if } \mathrm{Fr}<268
$$

$a=0.97$ if $F r \geq 268$

Where the Froude number $F r$ is given by:

$$
F r=\left[\frac{U^{2} \rho_{0}}{g d\left(\rho_{\infty}-\rho\right)}\right]^{1 / 2}
$$

\subsection{Governing equations}

The mathematical model of the problem is given by the conservation equations of Navier-Stokes written using the Reynolds decomposition which refers to the separation of flow variables (velocity $u_{i}$ and mass concentration $C^{\prime}$ ) into a mean (time averaged) component (mean velocity $\bar{u}_{i}$ in the directions $x_{i}$ and mean mass concentration $\bar{C}$ ) and a fluctuating component (fluctuating velocity $u_{i}^{\prime}$ and fluctuating mass concentration $C^{\prime}$ ):

$$
u_{i}=\overline{u_{i}}+u_{i}^{\prime}, \quad C=\bar{C}+C^{\prime}
$$

Thus, the mass, momentum and concentration equations for steady round horizontal Boussinesq buoyant jet can be written as:

Mass

$$
\frac{\partial \bar{u}_{i}}{\partial x_{i}}=0 \quad(i=1,2,3)
$$

\section{Momentum}

$\overline{u_{j}} \frac{\partial \overline{u_{i}}}{\partial x_{j}}=-\frac{1}{\rho_{r e f}} \frac{\partial \bar{p}}{\partial x_{i}}+\frac{\partial}{\partial x_{j}}\left(v \frac{\partial \overline{u_{i}}}{\partial x_{j}}-\overline{u_{i}^{\prime} u_{j}^{\prime}}\right)-\frac{\rho-\rho_{a}}{\rho_{a}} g$

Concentration

$$
\frac{\partial\left(\overline{u_{i} C}\right)}{\partial x_{i}}=\frac{\partial}{\partial x_{i}}\left(D \frac{\partial \bar{C}}{\partial x_{i}}-\overline{u_{i}^{\prime} C^{\prime}}\right)
$$

where $\overline{u_{i}}$ are the mean velocity components in the di- rections $x_{i}, \mu$ and $v$ are respectively the kinetic and the kinematic viscosities. $p$ is the pressure and $C$ is the the salt concentration of ambient fluid. $D$ is the coefficient of salt diffusion in fresh water. The turbulent stresses $\overline{u_{i}^{\prime} u_{j}^{\prime}}$ and the turbulent mass fluxes $\overline{u_{i}^{\prime} C^{\prime}}$ are unknown in the above equations and are approximated by the turbulence model.

\subsection{Turbulence model}

Turbulence model employed is the standard Averaged Reynolds-Number $k-\varepsilon$ model which turbulent kinetic energy $k$ and turbulence dissipation rate $\varepsilon$ equations are respectively:

$$
\begin{gathered}
\overline{u_{j}} \frac{\partial k}{\partial x_{j}}=v_{t}\left(\frac{\partial \overline{u_{i}}}{\partial x_{j}}+\frac{\partial \overline{u_{j}}}{\partial x_{i}}\right) \frac{\partial \overline{u_{i}}}{\partial x_{j}}+\frac{\partial}{\partial x_{j}}\left(\frac{v_{t}}{\sigma_{k}} \frac{\partial k}{\partial x_{j}}\right)-\varepsilon \\
\overline{u_{j}} \frac{\partial \varepsilon}{\partial x_{j}}=C_{\varepsilon 1} v_{t} \frac{\varepsilon}{k}\left(\frac{\partial \overline{u_{i}}}{\partial x_{j}}+\frac{\partial \overline{u_{j}}}{\partial x_{i}}\right) \frac{\partial \bar{u}_{i}}{\partial x_{j}}-C_{\varepsilon 2} \frac{\varepsilon^{2}}{k}+ \\
\frac{\partial}{\partial x_{j}}\left(\frac{v_{t}}{\sigma_{\varepsilon}} \frac{\partial \varepsilon}{\partial x_{j}}\right)
\end{gathered}
$$

where $v_{t}=C_{\mu} k^{2} / \varepsilon$ is the turbulent kinematic viscosity. The empiric constants $C_{\mu}, C_{\varepsilon 1}, C_{\varepsilon 2}, \sigma_{k}$ and $\sigma_{\varepsilon}$ which appear in Eqs.(12) and (13) are the constant standard Averaged-Reynolds-Number $k-\varepsilon$ model:

$$
C_{\mu}=0.09, C_{\varepsilon 1}=1.44, C_{\varepsilon 2}=1.92, \sigma_{k}=1, \sigma_{\varepsilon}=1
$$

It is known that the $k-\varepsilon$ model is based on hypotheses which are valid only for flows with high Reynolds numbers. In such flows, the predominance of viscous effects in a very thin area immediately through contact with the wall is negligible. However, the wall boundary represents a region where the velocity changes from the no-slip condition at the wall to its free flow value. The variation is usually the largest in the near-wall region, and hence strongest gradients are found here. Thus, one of the most common engineering problems is computing wall turbulent flows. Two remarkable effects of an adjacent wall are: damping the wall normal components, making the turbulent flow anisotropic and increasing the production of turbulence through the shearing mechanism in the flow.

In this work, to treat the boundary conditions in the presence of a wall boundary, friction forces are estimated by wall functions. These later resolve Eqs.(12) and (13) in the region of fully turbulence and out of 
boundary layer. Wall functions method is used to reduce computational requirement and increase numerical stability. This approach is based on a mesh, where the first interior node is located in the inertial sublayer (where the viscous effects are small), then it is possible to use the law-of-the-wall to specify the boundary condition for the dependent variables $u_{j}, k$ and $\varepsilon$. We note here that the near-wall region may be sub-divided into three different areas ${ }^{[15]}$.

viscous sub-layer: $0<y^{+}<5$,

buffer layer: $5<y^{+}<30$,

inertial sub-layer: $30<y^{+}<200$.

where $y^{+}$is a normalized value of the wall normal coordinate.

The standard wall function method is limited to flows where the turbulent kinetic energy becomes zero in separating and re-attachment points, by definition, where friction velocity $u_{\tau}\left(u_{\tau}=\sqrt{\tau_{\omega} / \rho}\right.$, where $\tau_{\omega}$ is the wall shear) is zero. Launder and Spalding models proposed a modification to the standard wall function method where they solve the turbulent kinetic energy with a modified integrated production and dissipation terms (Eq.(14)), and they set $\varepsilon$ with $k$ (Eq.15)).

$$
\begin{aligned}
& P_{k}=\frac{\tau_{p}^{2}}{\rho k C_{\mu}^{1 / 4} \sqrt{\kappa} E y} \\
& \varepsilon=\frac{C_{\mu}^{3 / 4} k^{3 / 2}}{\kappa y}
\end{aligned}
$$

where the subscript $p$ is used for the first interior node, $y$ is the wall normal coordinate, $k$ is the VonKarman constant, $E$ is the wall roughness coefficient, $\tau_{p}$ the wall shear stress.

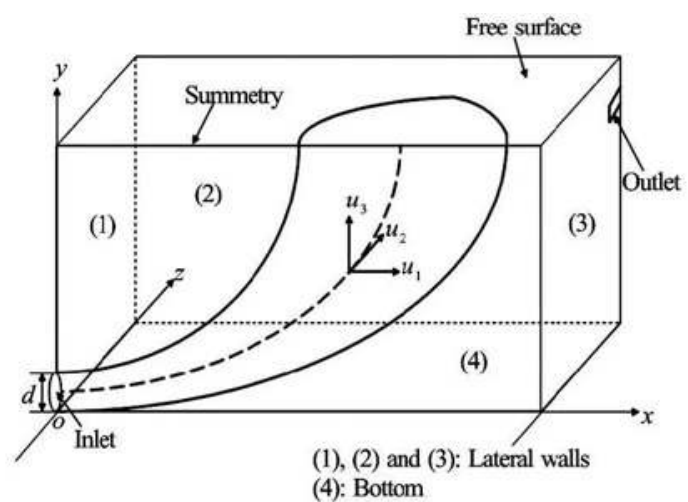

Fig.1 Scheme of coordinates system and boundary conditions

\subsection{Boundary conditions}

According to the symmetry of the injection no- zzle, the simulation computes only the half of jet domain. When solving the governing equations, appropriate initial conditions and boundary conditions (Fig. 1) needed to be applied. Hence, the following conditions were taken into account:

(1) The initial velocity of jet corresponds to the mean velocity of discharges flow rate. This condition is applied to compute mass flow into the domain and fluxes of momentum and concentration.

(2) Bottom and lateral walls of the tank correspond to a slip boundary condition which assumes that there is no viscous interaction between the wall and the fluid.

(3) Flow exits where the details of the flow velocity and pressure are not known prior to solution of the flow problem, are defined by "pressure outlet" condition. Latter, is appropriate where the exit flow is close to a fully developed condition, as the outflow boundary condition assumes a zero gradient for all flow variables except pressure.

(4) The free surface is designed by a symmetry boundary that indicates no penetration and vanishing shear stresses.

The following table summarizes the boundary conditions and $k-\varepsilon$ model conditions ${ }^{[16]}$ :

The computational domain (Fig.1) has been generated in tridimensional by about $2.2 \times 10^{6}$ of non-structured tetrahedral cells. To have more precision of numerical results, the mesh density at the nozzle and its surroundings was more refined than the rest of computational domain.

\begin{tabular}{|c|c|c|c|}
\hline $\begin{array}{l}\text { Boundary } \\
\text { condition }\end{array}$ & Velocity & $\begin{array}{c}\text { Turbulent } \\
\text { kinetic energy }\end{array}$ & $\begin{array}{c}\text { Dissipation } \\
\text { rate }\end{array}$ \\
\hline $\begin{array}{l}\text { Nozzle } \\
\text { section }\end{array}$ & $\begin{array}{c}\overline{u_{1}}=U_{0}, \\
\overline{u_{2}}=\overline{u_{3}}=0\end{array}$ & $\begin{array}{l}k=k_{0}= \\
10^{-3} v_{0}^{2}\end{array}$ & $\varepsilon=\frac{k_{0}^{3 / 2}}{0.5 d}$ \\
\hline Outlet & $\begin{array}{c}\frac{\partial \overline{u_{i}}}{\partial n}=0 \\
(i=1,2,3)\end{array}$ & $\frac{\partial k}{\partial n}=0$ & $\frac{\partial \varepsilon}{\partial n}=0$ \\
\hline $\begin{array}{l}\text { Bottom } \\
\text { and } \\
\text { lateral } \\
\text { walls }\end{array}$ & $\begin{aligned} \overline{u_{1}}=\overline{u_{2}} & = \\
\overline{u_{3}} & =0\end{aligned}$ & $\frac{\partial k}{\partial y}=0$ & $\frac{\partial \varepsilon}{\partial y}=0$ \\
\hline
\end{tabular}

Table 1 Generalized boundary and initial conditions

\subsection{Resolution method}

The resolution of steady governing equations in the presence of turbulence is done by the finite volume method with the solver Fluent-Ansys. The simulations were performed using 3-D segregated solver (pressure-based) and second order discretization. Implicit formulation was chosen. The SIMPLE algorithm was used for pressure-velocity coupling. The turbulence model used was the $k-\varepsilon$ Realizable with the full 
buoyancy effects.

Algebraic Equations for a discrete dependent variable $\Phi$ are constructed by integrating the governing equations over the separate control volumes. Hence, the result is given by

$$
A_{P} \Phi_{P}=\sum_{n b} A_{n b} \Phi_{n b}+s
$$

where indices $n b$ refer to adjacent cells to the cell $P$ and $A_{P}$ are coefficients including the convection and the diffusion terms of $\Phi$, whereas $S$ is a source term.

Convection terms are interpolated from the cell center values. This is accomplished using an upwind scheme. The diffusion terms are central differenced and this approach is known as the central-difference scheme second-order accurate.

The residual $R^{\Phi}$ is the imbalance in Eq.(10), which summed over all the computational cells $P$. This is referred to as the "unscaled" residual. It may be written as

$$
R^{\Phi}=\sum_{P}\left|\sum_{n b} A_{n b} \Phi_{n b}+s-A_{P} \Phi_{P}\right|
$$

To scale this residual, a scaling factor representative of the flow rate of $\Phi$ through the domain is used. Hence, the "scaled" residual is defined as

$$
R^{\Phi}=\frac{\sum_{P}\left|\sum_{n b} A_{n b} \Phi_{n b}+s-A_{P} \Phi_{P}\right|}{\sum_{P} A_{P} \Phi_{P}}
$$

The latter is a more appropriate indicator of convergence for most problems. At convergence, All discrete conservation equations (momentum, species, etc.) are obeyed in all cells to a specified tolerance or the solution no longer changes with subsequent iterations. To monitor convergence, Fluent uses a residual history. Generally, a decrease in residuals by three orders of magnitude indicates at least qualitative convergence. At this point, the major flow features should be established. Fluent recommends the value of $10^{-5}$ for concentration, $10^{-6}$ for energy and $10^{-3}$ for others quantities ${ }^{[17]}$. Starting from quiescent initial conditions, the equations are marched through time until converging to the steady-state solution. For each iteration, the momentum equations are solved using current values for pressure, to update the velocity field. The $k$ and $\varepsilon$ equations are solved using the previously updated values of the other variables. The fluid velocities are then updated, and a check for convergence of the system of equations is made. The steady-state solution is reached when the change in all of the calcula- ted variables is less than a chosen tolerance (residua$1 \mathrm{~s})^{[17]}$.

To minimize computation time, parallel calculation options have been set up by selecting Parallel under Processing Options in Fluent Launcher. Number of processes used is 4. Since our geometry involves enclosures connected via small-diameter nozzle injection, mean pressure levels in all zones can be quite large. Thus, the use of Double-precision calculations was necessary to resolve the pressure differences that drive the flow, since these will typically be much smaller than the pressure levels.

\section{Experimental model}

Numerical results obtained by the resolution of mathematical model are validating experimentally on a test bench in our laboratory. The experimental study of wall horizontal turbulent jet involves the behavior description, in the presence of a wall boundary, of fresh water jet injected into a homogenous and static ambient of salt water with different rates of salinity. Outgoing from the nozzle, the jet behavior is conditioned by: the nozzle diameter, initial velocity of injection and the initial density gradient between the jet and the ambient fluid.

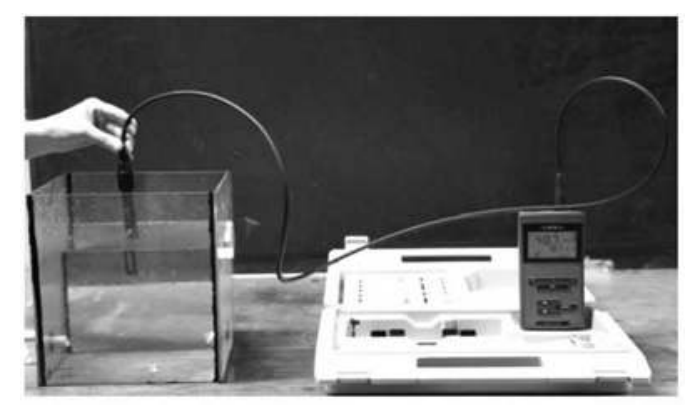

Fig.2 Mobile conductivity meter COND 3110 used to measure the salinity

Experiments are carried out on a plexiglas tank with a cross-section of $0.3 \mathrm{~m} \times 1 \mathrm{~m}$ and a depth of $0.3 \mathrm{~m}$. The tank is filled out by a static and homogenous ambient fluid of salt water. The salinity of this latter is deduced from the measure of solution conductivity by a mobile conductivity meter COND 3110 (Fig.2). This later is of a conductivity range of $0 \mu \mathrm{S}-1000 \mathrm{mS}$ with an accuracy of $\pm 0.5 \%$ full-scale \pm 1 LSD. Deduced salinities vary between $36 \mathrm{~g} / \mathrm{l}$ and $80 \mathrm{~g} / \mathrm{l}$.

Experimental apparatus is shown in Figs. 3 and 4. Fresh water, conducted by gravity into the tank, is injected horizontally from a circular nozzle with inner diameter ranging between $0.012 \mathrm{~m}$ and $0.004 \mathrm{~m}$. The flow of fresh water (colored by Indian ink) is controlled by a valve and measured by a flow meter which is capable of monitoring liquid fluids to $350 \mathrm{1} / \mathrm{min}$ with an accuracy of $\pm 0.5 \%$. The flow visualizations are 
carried out by a speed camera HS-650 TSI which captures at up to 636 frames per second and has a high resolution (1 280 $\times 1024$ Pixels) for water flows, lower speed air flows, and biological flows. Captured images are analyzed by the Image $\mathbf{J}$ software. This treatment is used to determine the shape of the plume and the corresponding coordinates.

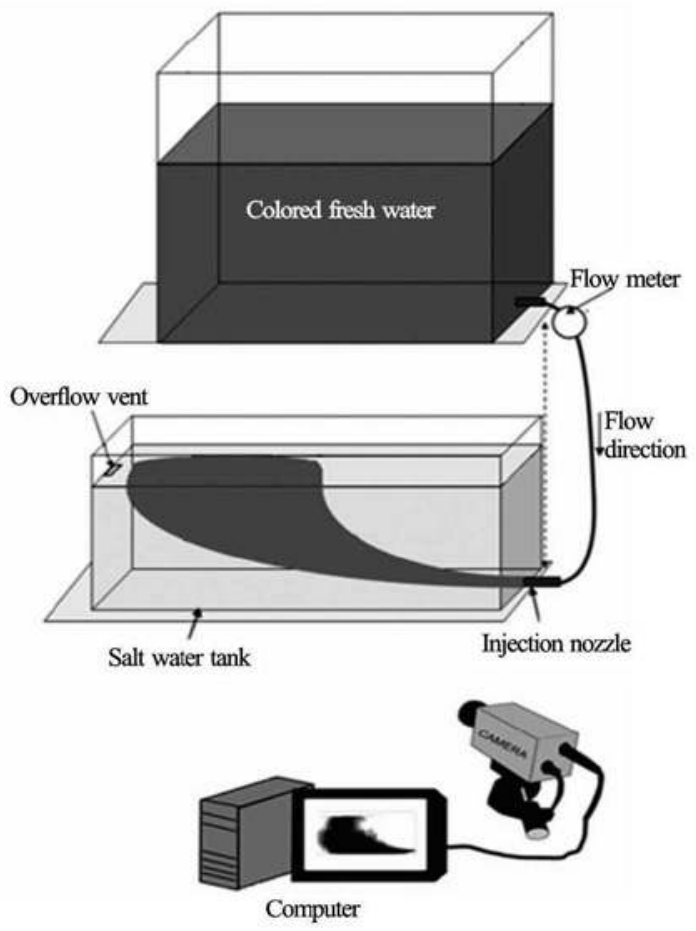

Fig.3 Scheme of experimental apparatus

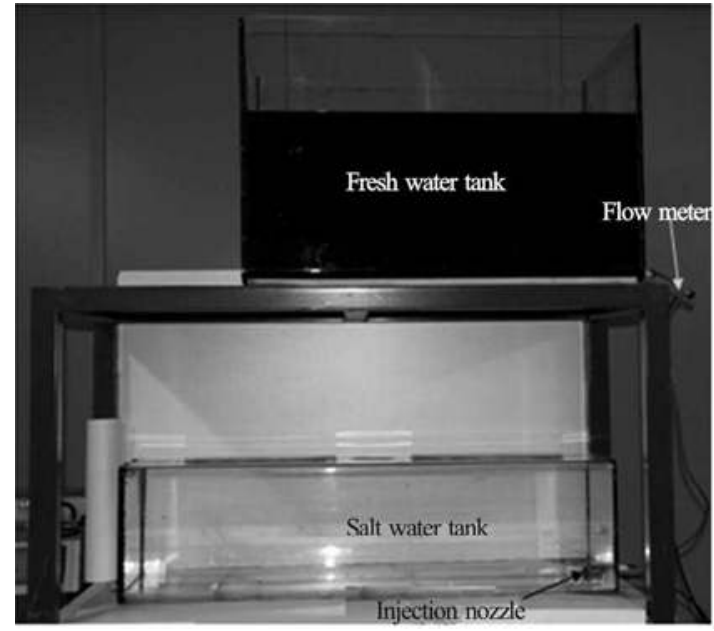

Fig.4 Experimental apparatus

In this work, experimental parameters are the nozzle diameter $d$, the flow rate of fresh water injected from the nozzle, and the mixture initial density which varies between densities of fresh and salt waters. Experiments have been carried out by varying these pa- rameters to cover the range of $2000-6000$ for initial Reynolds number and the range of 1-75 for initial Froude number. These dimensionless numbers are defined by

$\operatorname{Re}=\frac{U_{0} d}{v}, \quad F r=\left[\frac{U_{0}^{2} \rho_{\text {air }}}{g d\left(\rho_{\text {air }}-\rho_{\text {mixture }}\right)}\right]^{1 / 2}$

The following table summarizes the details of the experiments performed for each flow configuration:

Figures 5 and 6 show some experiments of Table 2 . Figure 5 presents the variation of flow behavior depending on salinity variation while the Fig. 6 shows the flow behavior depending on Reynolds number variation (variation of fresh water flow rate in the nozzle).

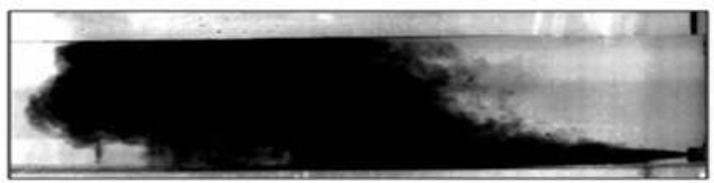

(a) With a salinity of $S=36 \mathrm{~g} /$

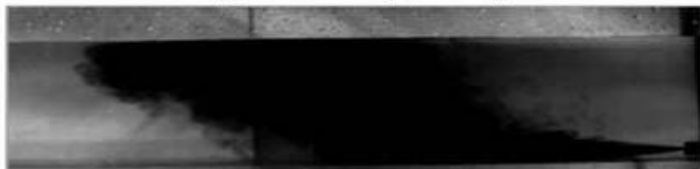

(b) With a salinity of $S=50 \mathrm{~g} /$

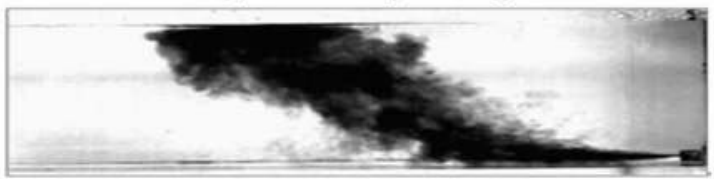

(c) With a salinity of $S=80.5 \mathrm{~g} /$

Fig.5 Experiments images of turbulent flow $(R e=5700)$ of fresh water jet into the tank of salt water

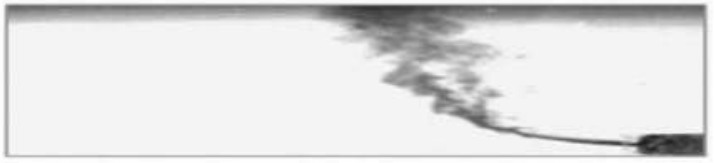

(a) $\operatorname{Re}=100$

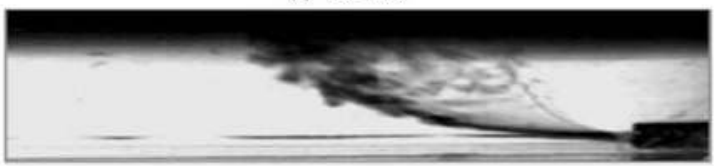

(b) $R e=183$

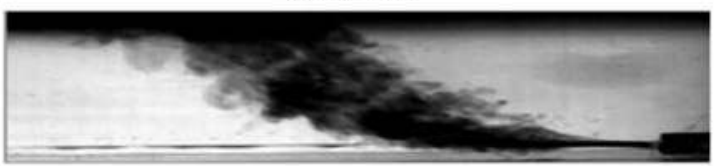

(c) $R e=385$

Fig.6 Experiments images of laminar flow of fresh water jet into the tank of salt water 
Table 2 Experiments details

\begin{tabular}{|c|c|c|c|c|c|c|}
\hline Experiment & $\begin{array}{l}\text { Velocity } \\
\left(U_{0} / \mathrm{m} / \mathrm{s}\right)\end{array}$ & Froude number $(F r)$ & Reynolds number $(R e)$ & $\begin{array}{l}\text { Salinity S of } \\
\text { ambient fluid }\end{array}$ & $\begin{array}{c}\text { Density } \\
\left(\rho_{0} / \mathrm{kg} \cdot \mathrm{m}^{-3}\right)\end{array}$ & $\begin{array}{c}\text { Density } \\
\left(\rho_{a} / \mathrm{kg} \cdot \mathrm{m}^{-3}\right)\end{array}$ \\
\hline 1 & 1.31 & 44 & 5700 & 36 & 1000 & 1027 \\
\hline 2 & 1.31 & 36 & 5700 & 50 & 1000 & 1038 \\
\hline 3 & 0.4 & 10 & 2000 & 50 & 1000 & 1038 \\
\hline 4 & 0.07 & 2 & 300 & 50 & 1000 & 1038 \\
\hline 5 & 0.025 & 0.6 & 100 & 50 & 1000 & 1038 \\
\hline 6 & 1.31 & 23 & 5700 & 80 & 1000 & 1062 \\
\hline 7 & 0.82 & 16 & 3300 & 80 & 1000 & 1062 \\
\hline 8 & 0.38 & 7 & 1400 & 80 & 1000 & 1062 \\
\hline 9 & 0.1 & 2 & 385 & 80 & 1000 & 1062 \\
\hline 10 & 0.05 & 1 & 183 & 80 & 1000 & 1062 \\
\hline
\end{tabular}

Qualitatively, the first step to validate mathematical model is to compare between numerical and experimental flow visualizations. Then, for a quantitative validation, those visualizations are translated into encrypted data and curves that describe the mixture density between plume and ambient fluid.

\section{Results and discussion}

\subsection{Jet boundaries}

The turbulent flow $(R e \geq 2000)$ pattern is generated by a continuous source of momentum and buoyancy. Numerical and experimental results are compared for boundaries and centerline trajectory. The initial conditions of the centerline quantities are taken as their values at the source. Since the flow is generated by both momentum and buoyancy, the rate between momentum and viscous forces is defined by the Reynolds number $R e$ while the Froude number $\mathrm{Fr}$ defines the rate between buoyancy and momentum. At the source, these numbers are defined by:

$$
R e=\frac{U_{0} d}{v}, F r=\left[\frac{U_{0}^{2} \rho_{a}}{g d\left(\rho_{a}-\rho_{0}\right)}\right]^{1 / 2}
$$

where $\rho_{0}$ is the fresh water jet density at the nozzle and $\rho_{a}$ is the density of ambient fluid (salt water).

In the following analysis, initial flow is represented by the initial velocity $U_{0}$ and the buoyancy by the initial gradient density $\Delta \rho_{0}=\rho_{a}-\rho_{0}$. During its rising as a result of buoyancy forces, the plume takes a curved form due to combined effect of the horizontal component of momentum and the gravity.

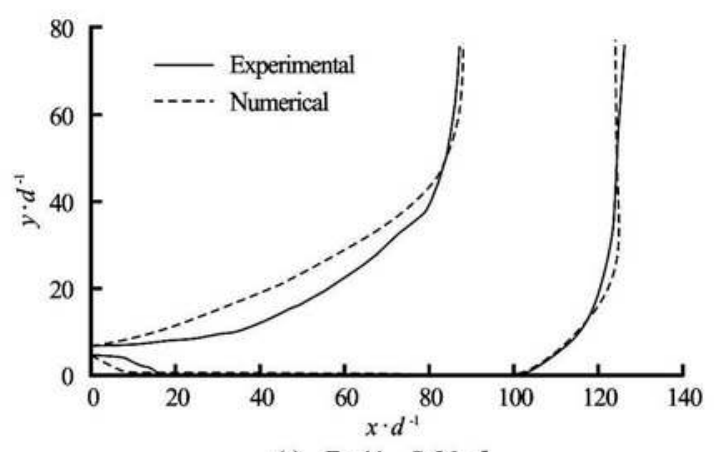

(a) $F r=44, S=36 \mathrm{~g} /$

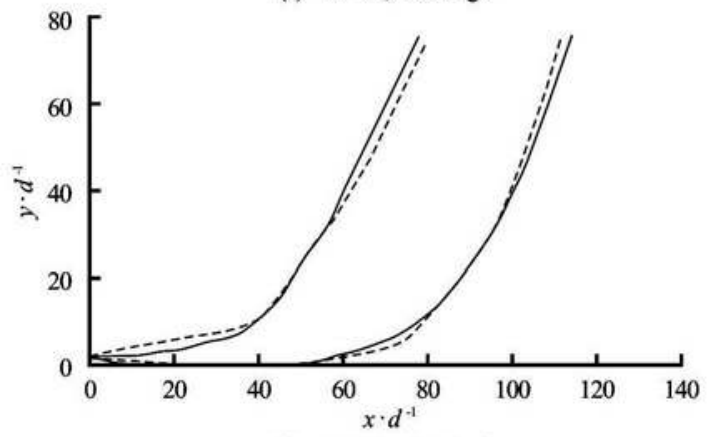

(b) $F r=36, S=50 \mathrm{~g} /$

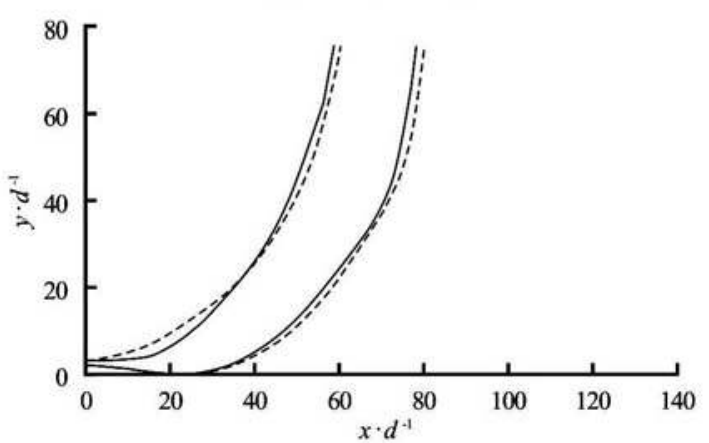

(c) $F r=23, S=80 \mathrm{~g} /$

Fig.7 Comparison of jet boundaries between numerical and experimental models for $U_{0}=1.31 \mathrm{~m} / \mathrm{s}$ and $R e=5700$ 
Outgoing from the nozzle, placed sufficiently close to the bottom, the jet seeps into the tank and the mixture process with ambient fluid starts. During its flow, the jet clings to the bottom for some distance. At the level of that boundary, the jet is submitted to frictional forces that brake the flow and decrease its kinetic energy. When this later becomes sufficiently weak, the flow is dominated by the positive buoyancy generated by initial density gradient $\Delta \rho_{0}$. Thus, the jet leaves the bottom and rises to surface as a free jet.

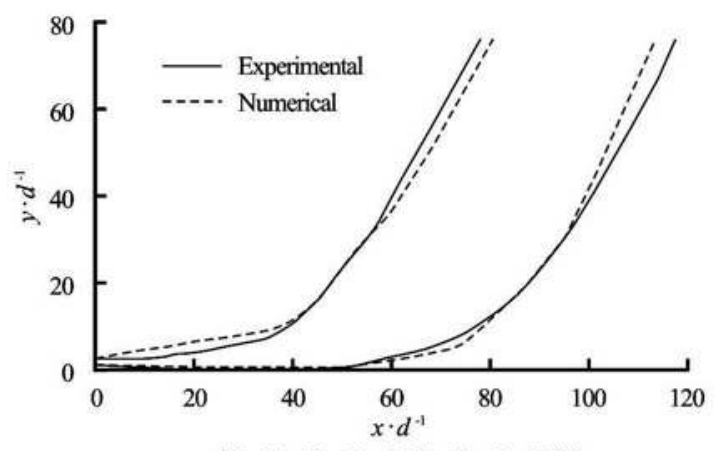

(a) $F r=36, U_{0}=1.31 \mathrm{~m} / \mathrm{s}, R e=5700$

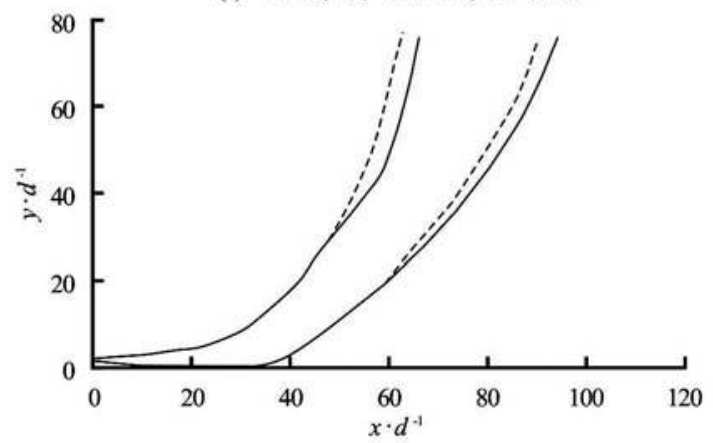

(b) $F r=10, U_{0}=0.4 \mathrm{~m} / \mathrm{s}, \operatorname{Re}=2000$

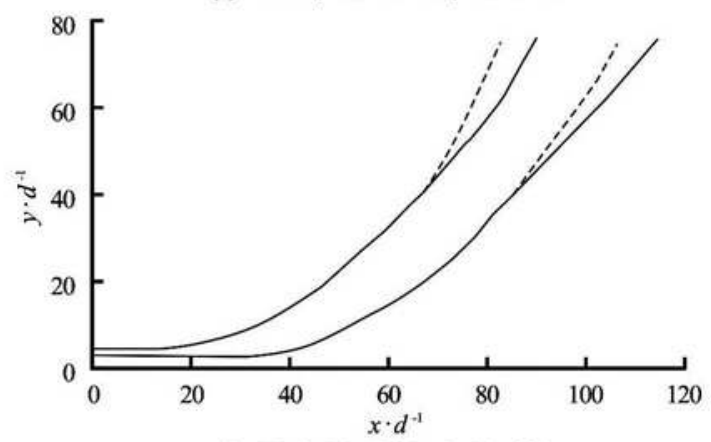

(c) $F r=2, U_{0}=0.07 \mathrm{~m} / \mathrm{s}, R e=300$

Fig.8 Comparison of jet boundaries between numerical and experimental models for an ambient fluid of $S=50 \mathrm{~g} / 1$

In principle, when a jet is injected horizontally from a nozzle with a certain momentum, it begins to ascend vertically as if the buoyancy forces predominates the inertia forces. However, in the case of horizontal jet of this study, for the same values of Froude number obtained by varying in the same time initial momentum flux, i.e., initial velocity $U_{0}$, and buoya- ncy flux, i.e., initial density gradient $\Delta \rho_{0}$ between mixture injection fluid and ambient fluid, the jet rising depends on $\Delta \rho_{0}$ rather than $U_{0}$. Even if the flow is generated by mixed convection, the buoyancy forces predominate: the higher these later are, i.e. low values of $\rho_{0}$, the faster and the nearest (to the nozzle) plume rising is (due to the important buoyancy) ${ }^{[18]}$. Thus, in this work we analyze the combined effect of turbulence and wall boundary on the behavior of a wall horizontal turbulent jet.

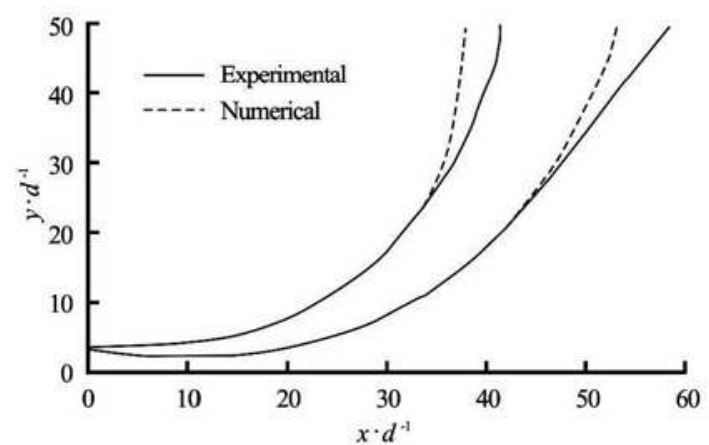

(a) $F r=7, U_{0}=0.38 \mathrm{~m} / \mathrm{s}, \operatorname{Re}=1400$

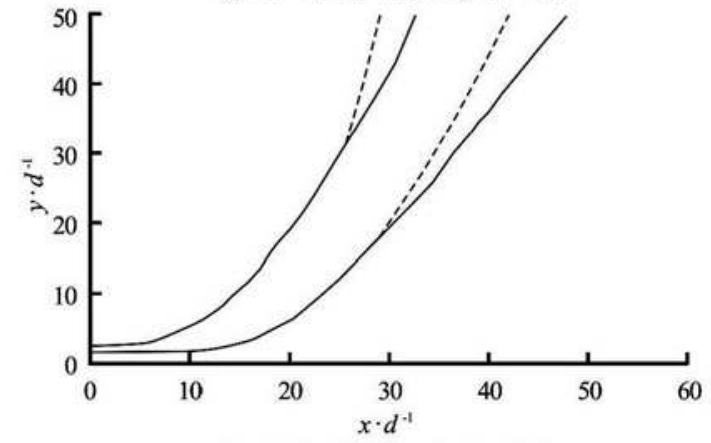

(b) $F r=2, U_{0}=0.1 \mathrm{~m} / \mathrm{s}, \operatorname{Re}=385$

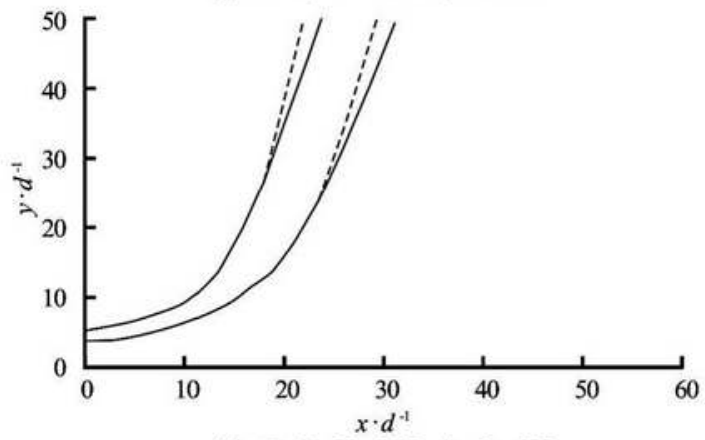

(c) $F r=1, U_{0}=0.05 \mathrm{~m} / \mathrm{s}, R e=183$

Fig.9 Comparison in laminar flow of jet boundaries between numerical and experimental models for an ambient fluid of $S=80 \mathrm{~g} / 1$

For a turbulent flow $(R e=5700)$, the fresh water jet is injected with the same initial velocity $U_{0}=1.31 \mathrm{~m} / \mathrm{s}$ into ambient fluids with different values of salt concentration $S=37 \mathrm{~g} / 1,50 \mathrm{~g} / 1$ and $80 \mathrm{~g} / 1$. We notice that the higher initial density gradient $\Delta \rho_{0}$ 
is (the smaller ambient fluid salinity $S$ is), the more important cling length $L$ is (Fig.7). In other words, the more Froude number decreases the more the attachment length to the wall is important.

Now, jets are injected in ambient fluids with a same salinity $S=50 \mathrm{~g} / 1$, corresponding to a same initial density gradient $\Delta \rho_{0}$, and where Froude numbers are obtained only by varying initial velocity $U_{0}$ (Fig.8). In this case, with the decrease of Froude number, the cling length decreases progressively in order to be zero when the flow becomes laminar.

To test the turbulence effect on the jet cling to the wall, we treat now the case of laminar flow for Reynolds numbers varying between 100 and 350 (Fig.9). In such flow, initial velocity $U_{0}$ at the source is very weak that the jet can't discharge too far over the nozzle. In this case, we notice that the wall boundary has no effect on the jet. This later, outgoing from the nozzle, raises up under the effect of buoyancy like a free jet.

For a wall turbulent jet injected from a source of momentum and positive buoyancy, the flow is conditioned by both turbulence effect and wall boundary that hits the jet. Jet cling to the wall depends strongly on the initial buoyancy flux where the lighter the jet is (more the initial density gradient is important), the weaker the jet cling to wall is. Indeed, when the jet is discharged from the nozzle, it merges with the ambient fluid under the effect of entrainment phenomena. However, for a light turbulent jet, entrainment processes is fast and the ambient fluid doesn't have the time to be entrained into the jet body. While this turbulent is combined to the positive buoyancy of the jet, this later raises up more rapidly when it is light. Thus, the jet doesn't cling to the wall for a long while. On the other hand, when the flow is laminar, the jet is discharged from the nozzle and guided by buoyancy effect with a behavior of free jet. In this case, the wall boundary has no effect on the jet flow.

In conclusion, the behavior of a wall horizontal turbulent jet depends on both initial Froude number $\mathrm{Fr}$ (that represents the ratio between buoyancy and momentum) and the effect of wall boundary (that the jet hit directly when it is discharged from the nozzle). Thus, this flow is characterized by its cling to the wall defined by a length $L$ called the cling length.

Numerical results for boundaries are compared with experimental results for each flow configuration described above. The improved numerical model predicts well the boundaries jet. Near the nozzle, where the flow is dominated by horizontal momentum, the agreement is very satisfactory, but when the flow becomes purely buoyant, numerical model presents some little deviation of jet boundaries. This is due es- sentially to the rate of ambient fluid entrainment into the jet body: in the near field, the entrainment rate $E$, given by relations 4 and 5 , is reduced to the component $E_{\text {mom }}$ (relation 6). This later depends only on the initial conditions which the values are known at the nozzle. That explains the accuracy of numerical results in the near field. When the jet leaves this later and arrives to the zone of established flow, the buoyancy dominates and the component $E_{\text {buoy }}$ (relation 7) takes part in the expression of entrainment rate $E$. That component introduces a new term $\theta$, inclination angle of jet trajectory, which is unknown in advance of numerical model. Thus, the perturbations detected are due to numerical resolution when the jet passes to buoyancy-dominated zone.
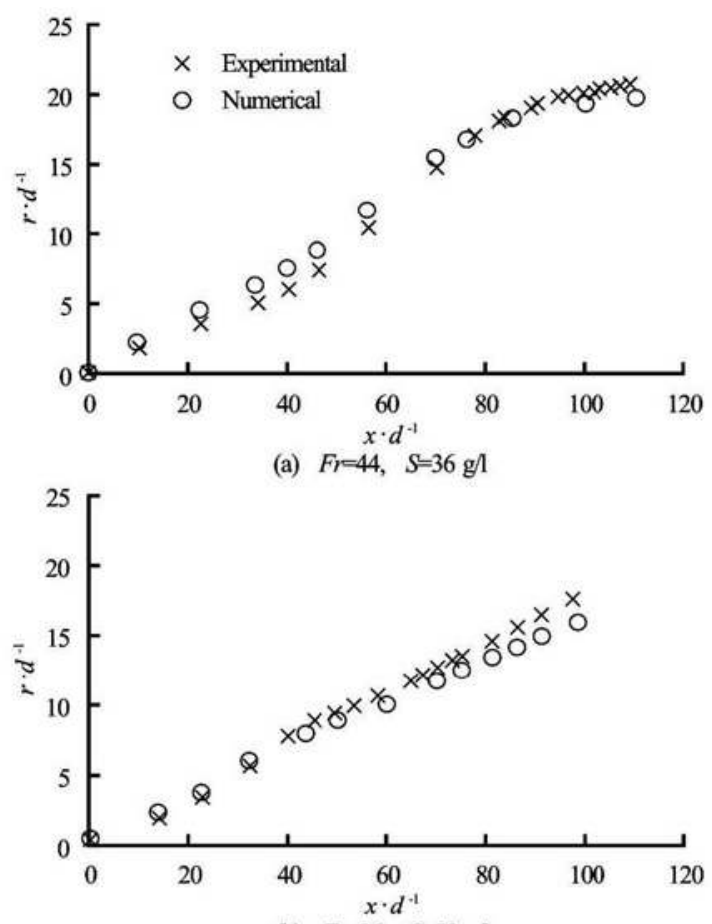

(b) $F r=36, \quad S=50 \mathrm{~g} /$

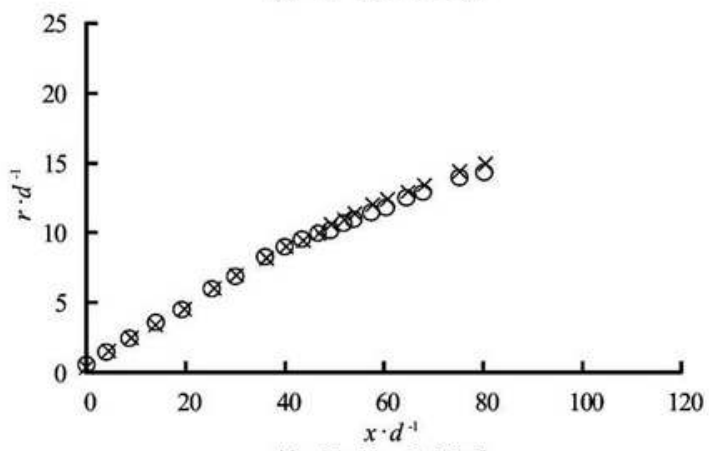

(c) $F r=23, S=80 \mathrm{~g} /$

Fig.10 Comparison of jet centerline radius between numerical and experimental models for $U_{0}=1.31 \mathrm{~m} / \mathrm{s}$ and $R e=5700$ 


\subsection{Jet centerline radius}

One of the most important characteristics to be determined for a flow jet is its radius $r$ or whether its half-width. This parameter quantifies the entrainment rate of the ambient fluid into the jet body: the more the entrainment is important, the more the jet becomes wider and $r$ increases. Thus, to continue the analysis of Section 3.1, we present in this section the variation of the jet radius $r$ on its centerline.

Jet centerline radius $r$ is determined and compared numerically and experimentally for each flow configuration described in Section 3.1.

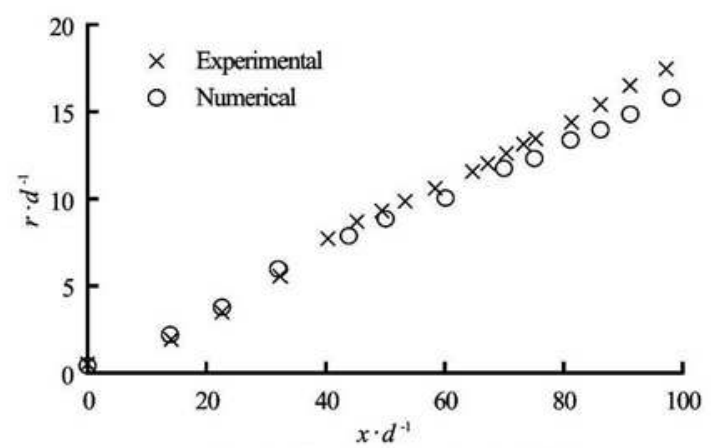

(a) $F_{r}=36, U_{0}=1.31 \mathrm{~m} / \mathrm{s}, R e=5700$

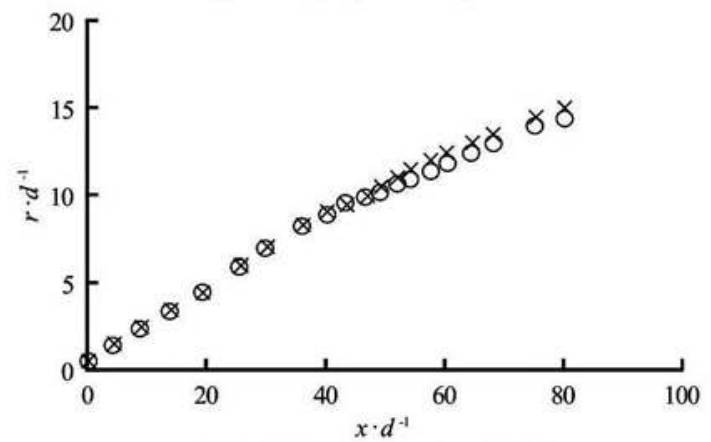

(b) $F r=10, U_{0}=0.4 \mathrm{~m} / \mathrm{s}, \operatorname{Re}=2000$

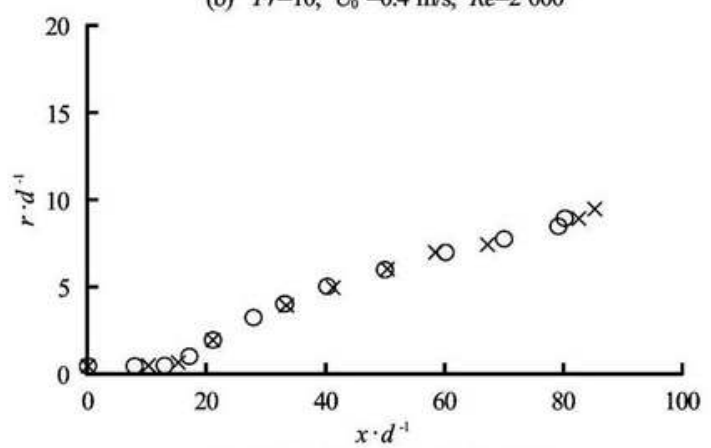

(c) Fr $=2, U_{0}=0.07 \mathrm{~m} / \mathrm{s}, \operatorname{Re}=300$

Fig.11 Comparison of jet centerline radius between numerical and experimental models for an ambient fluid of $S=$ $50 \mathrm{~g} / 1$

For each type of jet, round or plane, sample or double, free or with a wall boundary, with or without temperature (or density) gradient, the study of jet mean behavior in a static environment reveals that the mean velocity in the jet axis decreases and the jet ra- dius increases linearly (see for example Ref.[19]). Thus, for our case of wall horizontal turbulent jet and in accordance with the results of literature, the radius $r$ increases linearly. However, this increase depends strongly on the jet cling to the wall.
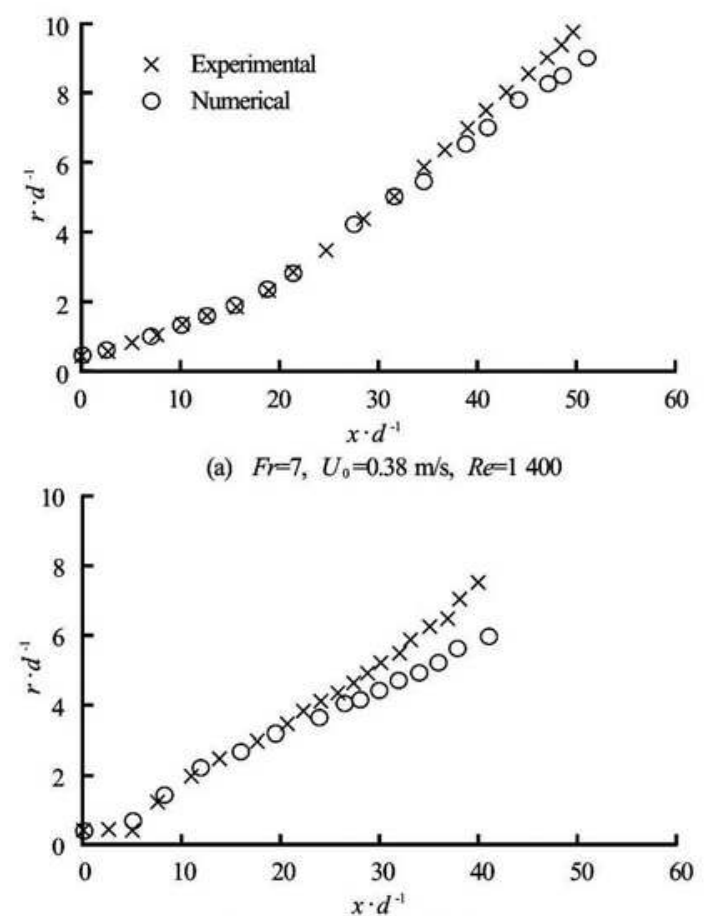

(b) $F r=2, U_{0}=0.1 \mathrm{~m} / \mathrm{s}, R e=385$

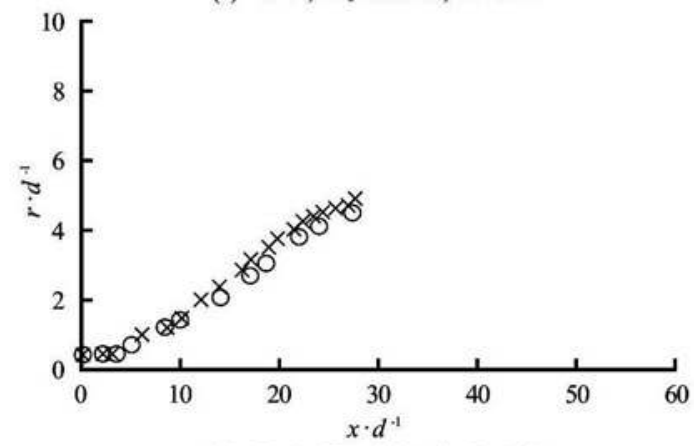

(c) $F r=1, U_{0}=0.05 \mathrm{~m} / \mathrm{s}, \operatorname{Re}=183$

Fig.12 Comparison of jet centerline radius between numerical and experimental models for an ambient fluid of $S=$ $80 \mathrm{~g} / 1$

For turbulent flows of the same initial Reynolds number $(R e=5700)$ and the same initial velocity $\left(U_{0}=1.31 \mathrm{~m} / \mathrm{s}\right)$, the radius $r$ increases linearly and the jet expansion is more important for the flows of high values of Froude number (Fig.10). These later, correspond in the same time to the cases where the cling to the wall is important. Indeed, in the near field, these jets have the same behavior and keep practically the same width. However, from the moment where the inertia force damps and becomes equal to zero under the effect of friction with the wall, the jet behavior 
changes and becomes dominated by buoyancy. While this later isn't the same for those jets, in the far field the radius evolution changes: the lighter the jet (high values of $\Delta \rho_{0}$ ) is, the weaker its expansion is.

Proceeding with environments of the same salinity $S$, the density gradient $\Delta \rho_{0}$ is the same for flows of different values of Froude number $F r$, initial velocity $U_{0}$ and Reynolds number $R e$. Jet radius at the extreme point of centerline trajectory is more important for high values of Froude number $\mathrm{Fr}$ and decreases with the decrease of Reynolds number $R e$ (passing from turbulent to laminar flow) (Fig.11). Indeed, more the flow is turbulent, more it entrains ambient fluid. This explains the decrease of radius $r$ when we pass to laminar flow. To confirm this deduction, we analyze in the next section, the evolution of jet radius for flows purely laminar.

For laminar flows, jet inertia forces are very weak to entrain high quantities of ambient fluid into the jet body. Thus, the entrainment rate and jet expansion are weak. However, the flow is purely buoyant where the radius $r$ decreases with the decrease of Reynolds number $\operatorname{Re}$ (Fig.12).

\subsection{Jet cling length to the wall}

According to the previous analysis, the cling of a turbulent buoyant wall jet injected from a nozzle placed sufficiently near a wall depends, at the same time, on inertia forces and buoyancy forces. The flow is especially characterized by the Froude number $F r$.

Near the wall, the mixture of the jet with the ambient fluid is defined by a length called the mixture length. This later is the product of the cling distance to the wall and the Von Karman constant (determined experimentally). This relation represents a law that leads to the identification of logarithmic profiles to the velocity near the wall ${ }^{[20]}$. However, an ambiguity remains always to define the distance travelled by the jet on the wall. Thus, we define this region by the cling length $L$ caused by Coanda effect.

Near the wall region, the buoyant turbulent jet is injected with an important momentum flux that generates a pressure difference between the jet and the wall. In the near field (potential core), the inertia forces of the jet dominate the flow and lead the jet to cling to the wall under the effect of pressure difference. With the wall friction, the jet velocity decreases and becomes equal to zero. When the pressure forces become weak in comparison with the buoyancy forces, the jet leaves the wall and raises up vertically like a free jet. This point of detachment represents the limit of the cling length $L$. On this subject, the study of Ref.[2] introduced, analytically and experimentally, the relations between the cling length $L$ of the wall jet and the Froude number. Recently, Huai et al. ${ }^{[3]}$, have compared their numerical results for the jet cling length with the results of Sharp.

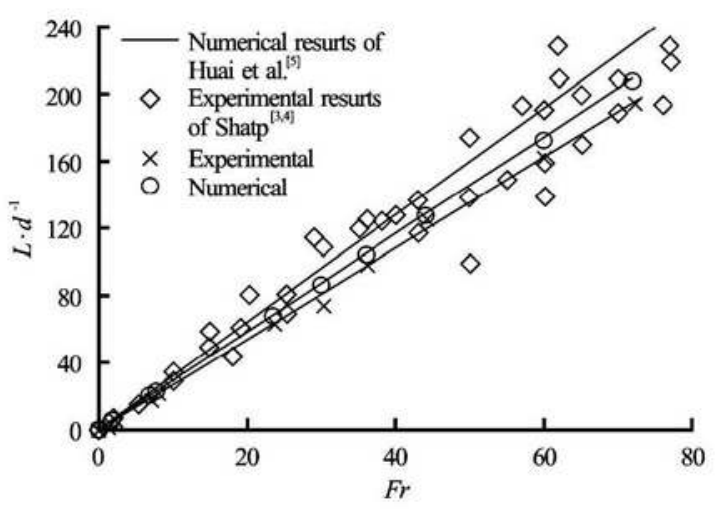

Fig.13 Comparison of the numerical and experimental results with the literature for the variation of the jet cling length $L$ in function of initial Froude number

Here, we compare our numerical and experimental results with the literature, especially with those of Sharp and Huai et al. (Fig.13). The jet cling to the wall under the Coanda effect is related to Froude number $F r$ by a linear law that shows the increase of cling length $L$ with the increase of Froude number. According to Ref.[3], this law is given by: $L / d=3.2 F r$. The comparison of our numerical and experimental results with the experiments of Sharp shows a very satisfactory agreement.

\section{Conclusions}

In this paper, a numerical and experimental investigation of Boussinesq wall horizontal turbulent jet of fresh water in a static homogeneous environment of salt water is considered. Mathematical and experimental models have been used to determine jet boundaries, centerline jet radius and jet cling length to the wall. The solutions of jet behavior will be useful not only to the marine disposal problems but also to the problems of a similar nature such as the disposal of hot waste gases into the atmosphere or hot water from a thermal power plant into a lake.

Several flow configurations are obtained by varying initial injection conditions: nozzle diameter, injection velocity and density gradient between the jet and the ambient fluid (salt water). Mathematical approach is based on numerical resolution of conservation equations of mass, momentum and concentration, and turbulence equations by Reynolds Averaged Navier Stokes finite volume method. Numerical results are validated by comparison with experimental results (which apparatus was described in sect. 3) and a good level of agreement is observed.

The analysis focus essentially on the influence of the combined effect of turbulence and wall boundary on the jet behavior, especially its cling to the wall. In dimensionless values, numerical and experimental de- 
termine the boundaries, centerline radius and cling length of the jet to the wall.

Results show that the flow is dominated by buoyancy forces. Indeed, the jet cling to the wall, under the Coanda effect, depends strongly on the initial buoyancy flux: the lighter the turbulent jet is, the weaker its cling to the wall is. However, contrary to the case of free buoyant turbulent jet ${ }^{[18]}$, the turbulence effect influences remarkably the jet behavior. Thus, there is no jet cling to the wall for laminar flow. In this later, the flow is purely buoyant since the source and the wall has no effect on the jet.

During its development, the jet entrains the ambient fluid. Entrainment process promotes the jet expansion and increases its radius (half-width). In accordance with literature, our results show that the jet radius increases linearly and the jet expansion increases with the increase of initial Froude number. In others words, more the jet clings to the wall, for high values of Froude number $\mathrm{Fr}$, more the jet becomes wide with an important entrainment of ambient fluid. Thus, a good jet dilution is established.

According to these analysis, a good concordance is established between the jet cling to the wall and the dependence of the flow on the ratio between inertia and buoyancy forces (represented by Froude number $F r$ ). Thus, a linear law is defined between this later and the jet cling length $L$, thus helping to delimit the mixture zone of a wall jet.

\section{References}

[1] SINGH N. K., RAMAMURTHI K. Formation of Coanda jet from sharp-edged swirl nozzle with base plate $[\mathrm{J}]$. Experimental Thermal and Fluid Science, 2009, 33(4): 675-682.

[2] SHARP J. J., VYAS B. D. The buoyant wall jet[J]. Ice Proceedings, 1977, 63(3): 593-611.

[3] HUAI Wen-xin, LI Zhi-wei and QIAN Zhong-dong et al. Numerical simulation of horizontal buoyant wall jet[J]. Journal of Hydrodynamics, 2010, 22(1): 58-65.

[4] LI Z., HUAI W. and QIAN Z. Large eddy simulation of flow structure in the near region of a circular wall jet[J]. Procedia Engineering, 2012, 31: 713-722.

[5] ZEYTOUNIAN R. K. Joseph Boussinesq and his approximation: A contemporary view[J]. C. R. Mécanique, 2003, 331: 575-586.

[6] BOIS P. A. Joseph Boussinesq (1842-1929): A pioneer of mechanical modelling at the end of the 19th Century[J]. C. R. Mécanique, 2007, 335: 479-495.
[7] EL-AMIN M. F. Non-Boussinesq turbulent buoyant jet resulting from hydrogen leakage in air[J]. International Journal of Hydrogen Energy, 2009, 34(18): 78737882.

[8] SWAIN M. R., FILOSO P. and GRILLIOT E. S. et al. Hydrogen leakage into simple geometric enclosures[J]. International Journal of Hydrogen Energy, 2003, 28(2): 229-248.

[9] VANIERSCHOT M., BULCK E. V. Hysteresis in flow patterns in annular swirling jets[J]. Experimental Thermal and Fluid Science, 2007, 31(6): 513-524.

[10] CHIEKH M. B., BERA J. C. and SUNYACH M. Synthetic jet control for flows in a diffuser: Vectoring, spreading and mixing enhancement $[\mathrm{J}]$. Journal of Turbulence, 2003, 4(5): 37-41.

[11] JIANG H., BREIER J. A. Physical controls on mixing and transport within rising submarine hydrothermal plumes: A numerical simulation study[J]. Deep Sea Research Part I Oceanographic Research, 2014, 92(10): 41-55.

[12] FAN L. N., BROOKS N. H. Discussion of Ref. 2[C]. Proceeding of ASCE Journal of Hydrahlic Division. 1966, HY2: 423-429.

[13] HOUF W. G., SCHEFER R. W. Analytical and experimental investigation of small-scale unintended releases of Hydrogen[J]. International Journal of Hydogen Energy, 2008, 33: 1435-1444.

[14] EL-AMIN M. F., SUN S. and KANAYAMA H. NonBoussinesq turbulent buoyant plume of a low-density gas leaks into a high-density ambient[J]. Applied Mathematics and computation, 2010, 217(8): 3764-3778.

[15] TENNEKES H., LUMLEY J. L. A first course in turbulence[M]. Cambridge, USA: The MIT Press, 1972.

[16] MEHTA D., Van ZUIJLEN A. H. and KOREN B. et al. Large eddy simulation of wind farm aerodynamics: A review[J]. Journal of Wind Engineering and Industrial Aerodynamics, 2014, 133: 1-17.

[17] ANSYS FLUENT. Tutorial guide[R]. 2009.

[18] BELCAID A. Modélisation de la dispersion des pollutants dans un milieu marin via les oueds et les émissaires sous-marins[D]. Application to the pollution of the Bay of Tangier (Morocco), Doctoral Thesis. Marseille, France: Aix-Marseille Universitys, 2013, 183(in France).

[19] HAN G., ZHOU M. D. and WYGNANSKI I. Some observations of the coanda effect. engineering turbulence modelling and experiments $6[\mathrm{C}]$. Procedings of the ERCOFTAC International Symposium on Engineering Turbulence Modelling and Measurements, ETMM6. Sardinia, Italy, 2005, 545-553.

[20] THUAL O. Hydrodynamics of the environment[M]. Paris, France: Ecole Polytechnique, 2010, 328(in France). 